University of Nebraska - Lincoln

DigitalCommons@University of Nebraska - Lincoln

Mammalogy Papers: University of Nebraska

State Museum

Museum, University of Nebraska State

6-1-2008

\title{
Material Properties of Coyote Dentine under Bending: Gradients in Flexibility and Strength by Position
}

\author{
Patricia W. Freeman \\ University of Nebraska-Lincoln, pfreeman1@unl.edu \\ Cliff A. Lemen \\ University of Nebraska-Lincoln, clemen2@unl.edu
}

Follow this and additional works at: https://digitalcommons.unl.edu/museummammalogy

Part of the Zoology Commons

Freeman, Patricia W. and Lemen, Cliff A., "Material Properties of Coyote Dentine under Bending: Gradients in Flexibility and Strength by Position" (2008). Mammalogy Papers: University of Nebraska State Museum. 110.

https://digitalcommons.unl.edu/museummammalogy/110

This Article is brought to you for free and open access by the Museum, University of Nebraska State at DigitalCommons@University of Nebraska - Lincoln. It has been accepted for inclusion in Mammalogy Papers: University of Nebraska State Museum by an authorized administrator of DigitalCommons@University of Nebraska Lincoln. 
Published in Journal of Zoology 275:2 (June 2008), pp. 106-114; doi 10.1111/j.1469-7998.2008.00411.x

Copyright (C) 2008 P. W. Freeman and C. A. Lemen; journal compilation (c) 2008 The Zoological Society of London; published by

Blackwell Publishing. Used by permission.

Submitted October 1, 2007; accepted December 17, 2007.

\title{
Material properties of coyote dentine under bending: Gradients in flexibility and strength by position
}

\author{
P. W. Freeman and C. A. Lemen
}

School of Natural Resources and University of Nebraska State Museum, University of Nebraska-Lincoln

Correspondence: Patricia W. Freeman, School of Natural Resources and University of Nebraska State Museum, University of Nebraska-Lincoln, 428 Hardin Hall, Lincoln, NE 68583-0974, USA; Email: pfreeman1@unl.edu

\begin{abstract}
We investigate the nonlinear properties of dentine from the upper canines of coyotes Canis latrans with bending tests. With the results we predict the behavior of whole canines under load. Coyote dentine is not homogeneous but is stronger and more ductile farther away from the pulp cavity. The modulus of rupture (MOR) first increases and then declines with distance from the pulp cavity. Our analysis of the composite nature of dentine produced by these gradients indicates that there may be an adaptive explanation with the composite having nearly the strength of the strongest dentine and a work of fracture greater than even the most ductile dentine. Coyote dentine is considerably stronger than human dentine. In coyotes, the peak MOR, a measure of bending strength, is $480 \mathrm{MPa}$, compared with a maximum of $225 \mathrm{MPa}$ for human dentine. This value is about the same as the weakest coyote dentine that we found near the pulp cavity. Finally, enamel plays, at most, a small role in the bending strength of the whole tooth. Our results indicate that enamel under tension adds little to strength, but we cannot dismiss a small role for enamel in compression.
\end{abstract}

Keywords: dentine, enamel, canine tooth, tooth strength, finite element analysis, Canis latrans

\section{Introduction}

Previously, we studied the strength of whole teeth by measuring the force needed to break them in bending (Freeman \& Lemen, 2007). We calculated ultimate stress using finite element analysis (FEA) with a linear model of the stress-strain curve. We were able to produce an accurate predictive model for the strength of canines that treated dentine and enamel as a single linear homogeneous material. Our model was predictive of whole tooth behavior, but not descriptive of stresses and strains that actually occurred within the tooth because of limitations of our linear assumption (Currey, 2002). Here we continue our work by quantifying the material properties of dentine and allowing for nonlinearity and gradients of material properties within the tooth (Figure 1).

Dentine is not a homogeneous material; within one tooth, material properties such as ultimate tensile stress (UTS) and ultimate tensile strain vary depending on position (for human dentine, see Kishen, Ramamurty \& Asundi, 2000; Staninec et al., 2002; Imbeni et al., 2005). We quantify properties of dentine in the upper canine teeth of the coyote Canis latrans to determine whether differences in dentine exist and how this variation within the tooth may impact tooth strength.

There are several aspects to tooth strength such as resistance to wear, compressive strength to axial loads, shearing strength, tensile strength, and bending strength. Each aspect of tooth strength requires different experimental approaches for study. Here we concentrate on the bending strength of canine teeth. Although we understand that other aspects of tooth strength may be important, we needed to narrow our focus to make a more manageable project. Our desire to look at bending strength springs from our intuition that bending stresses may cause canine teeth to break in wild carnivores. The aggressive use of canines in carnivores will generate large forces, and the length of these teeth combined with large forces will cause high bending moments. Van Valkenburgh (1988) found tooth breakage to be common in large carnivores and the canines were the most commonly broken teeth.

In previous work, we used a simple linear model for the behavior of dentine. Here we extend our analysis to a nonlinear model. Although we argue that the bending method used here is biologically relevant, quantification of the nonlinear nature of dentine in a bending test is problematic. Nonlinear FEA (bilinear model, Figure 2) allows these nonlinear properties to be partially quantified. The bending test is performed and the displacement to force curve is recorded. Using FEA we can determine what the bilinear properties of a material would be to produce this kind of displacement to force curve. In Figure 2 the lower curve (a) represents the observed data based on the bending experiment with a dentine sample. These data are used to estimate the bilinear properties (Figure 2, curve b). 


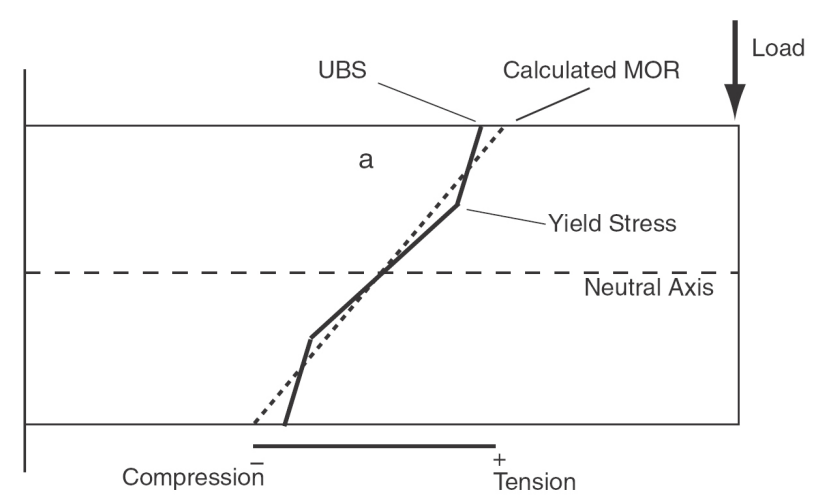

Figure 1. A cantilevered beam attached at the left with distribution of tensile and compressive stresses along line a superimposed upon it. The beam is made of a nonlinear material and has been loaded beyond linear response to the breaking point. The distribution of stresses is shown as a solid curve. Once the yield stress is reached, the stress-strain relationship is altered. The ultimate bending stress (UBS) is the actual maximal stress experienced. Based on the load and dimension of the beam, the distribution of stresses can be calculated by assuming a linear model and are shown as the dashed line. Under this linear model the extreme tensile stress at failure is called the modulus of rupture (MOR). While the MOR is useful as a simple index of strength, this stress is not experienced in the beam.

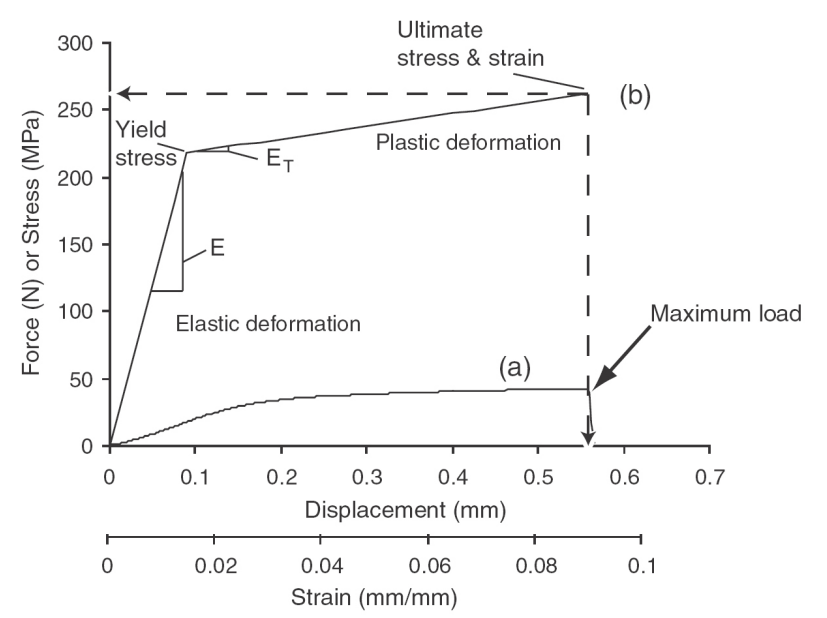

Figure 2. The result of a three-point breaking experiment is shown here as curve a. Using finite element analysis with a bilinear model the values of Young's modulus $(E)$, yield stress, post-yield modulus $\left(E_{\mathrm{t}}\right)$, ultimate bending stress, and ultimate strain can be calculated (curve b). All of these values are determined in a bending experiment and are not applicable to breaking in simple tension.

\section{Materials and methods}

\section{Source material}

Canine teeth of coyotes were collected from animals killed in predator control programs in Nebraska. Fresh teeth were refrigerated in saline solution until use within a few months of death. A human canine tooth was also used for dentine samples. This tooth had been preserved in a $1 \%$ thymol solution for an unknown length of time.

\section{Sample preparation}

Using a diamond saw (Isomet Slow Speed Saw, Buehler Worldwide Headquarters, Lake Bluff, IL, USA) columns about $0.90 \times 0.90 \times 13 \mathrm{~mm}$ were cut from the canine teeth. Using this saw three kinds of columns were cut. First, the long-axis columns were cut parallel to the long axis of the tooth. Second, the short-axis columns paralleled the anterior-posterior (mesial-distal) base of the tooth. Finally compression columns were cut with their long axis parallel to the long axis of the tooth, but these columns were cut with lengths of only $1 \mathrm{~mm}$ to avoid Euler bending problems in the compression tests.

To make long-axis columns, teeth were mounted in die stone, and a series of parallel cuts was made at an interval of about $0.90 \mathrm{~mm}$. Then the tooth was rotated $90^{\circ}$, and a second series of parallel cuts were made. This produced a matrix of columns still attached at their bases. Soft putty was used to maintain the position of these columns while the tooth was turned for the final cut to free the columns from the base of the tooth. Columns were then numbered and stored individually in saline solution and refrigerated.

\section{Tension, bending, and compression fixtures}

Bending strength was found using a three-point bending fixture with the two ends of the column simply supported (Figure 3). The indenter was driven downward at a rate of $0.1 \mathrm{~mm} \mathrm{~s}^{-1}$ by the Inspec 2200 (Instron Corp., Norwood, MA, USA) and the displacement to force measurements

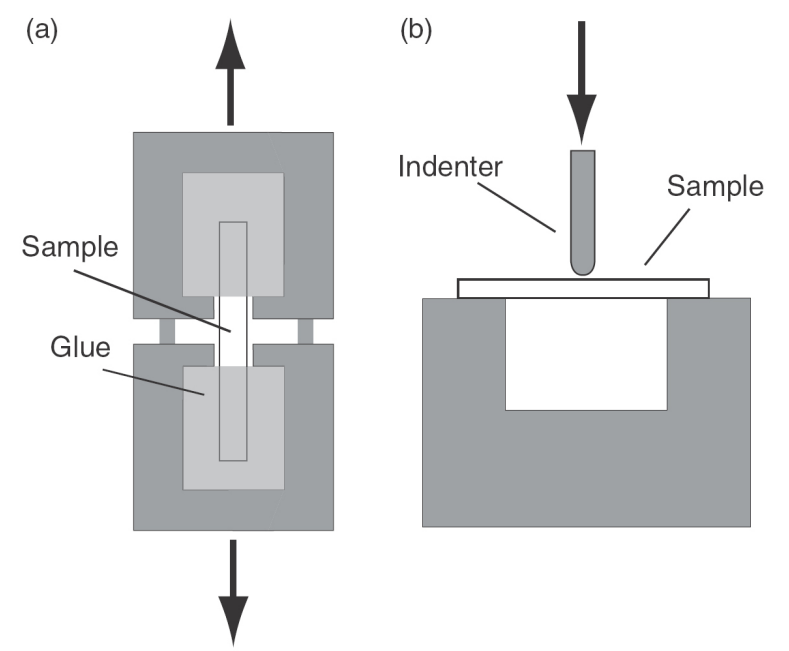

Figure 3. Two methods for determining two types of ultimate stress. In (a) is the tension test that is often considered the classic way to determine ultimate tensile stress (UTS). In (b) is a three point rupture test that breaks a simply supported beam. This test can be used to calculate the modulus of rupture (MOR) or with the aid of FEA, the ultimate bending stress (UBS). 
were recorded. The modulus of rupture (MOR) was calculated as

$$
\mathrm{MOR}=1.5\left(P_{\max } l\right) / b d^{2}
$$

where the maximum load is $P_{\text {max }} l$ is the span of the beam, $b$ equals the width of the rectangular beam, and $d$ is the depth of the beam. Equation (1) assumes that the stressstrain relationship right up to failure is linear (Currey, 2002).

To find compressive strength the sample was placed on a flat aluminum block and crushed by a flat indenter mounted in the Inspec. Compressive strength was calculated as maximum load divided by the cross-sectional area of sample.

For the tension fixture the column of dentine was glued into place with cyanoacrylate glue (Figure 3). Then the fixture was mounted into the Inspec and pulled apart at $0.1 \mathrm{~mm} \mathrm{~s}^{-1}$. This fixture was problematic because the dentine often slipped out of the glue before it broke. Also strain in the glue may well have confounded efforts to calculate strain and Young's modulus in the dentine. In our view this setup is useful only for the calculation of UTS, which is

$$
\mathrm{UTS}=P_{\max } / A
$$

where $A$ is the cross-sectional area of the neck of the sample where failure occurs.

\section{Breaking whole teeth}

Using methods of Freeman \& Lemen (2007), whole teeth were mounted onto a small length of copper pipe with die stone. When die stone sets it becomes a stony material that is ideal for this task. Imbedded canines were broken with an Instron testing machine (Instron Corp.) by applying force to the posterior edge of the tooth with a steel indenter at a point $70 \%$ of the tooth's length from the level of embedding. The speed of loading was set at $1 \mathrm{~mm} \mathrm{~min}^{-1}$. To avoid large stresses where the indenter meets the tooth, we inserted a leather pad (thickness $=1.6 \mathrm{~mm}$ ) to spread the load.

To help determine the role of enamel in tooth strength, we ground the enamel away completely on some teeth (whole grind) and from the posterior half only of other teeth (half grind). The enamel was removed using a small abrasive wheel mounted in a high-speed rotary tool. These teeth were taken as pairs from one animal with one tooth being ground and the other not. This gave us a control to compare the breaking strength of the tooth with and without grinding.

\section{FEA}

Models of teeth were created in Rhinoceros CAD program (Version 3, Robert McNeel \& Associates, Seattle, WA, USA) to produce a three-dimensional model of the tooth (Freeman \& Lemen, 2007). The tooth models were imported into FEMPRO (Version 20.1, Algor, Pittsburgh, PA, USA) for a bilinear analysis. Because of the simple structure of the dentine columns, they were modeled within FEMPRO us- ing the CAD capabilities of this program as a 2-D element with a mesh size of $c .0 .15 \mathrm{~mm}$. The material model was von Mises with isotropic hardening and analysis formation was total Lagrangian. The fixture was also modeled so that the rupture experiments could be analyzed in FEMPRO.

Armed with the correct dimensions of a column used in a real rupture experiment, we could repeat the experiment within FEA and measure the displacement to force curve obtained in the virtual experiment. When using a bilinear model, there are three parameters that describe a material's behavior (Figure 2): Young's modulus ( $E$, the slope of the stress to strain curve during the initial linear and elastic phase of deflection), yield stress (stress where plastic deformation begins and the stress-strain curve changes slope), and the post-yield modulus ( $E_{t^{\prime}}$ the slope of the stress-strain curve after the yield point). Altering these three parameters alters the displacement to force curve from the FEA analysis. It is a simple matter to fit the virtual displacement
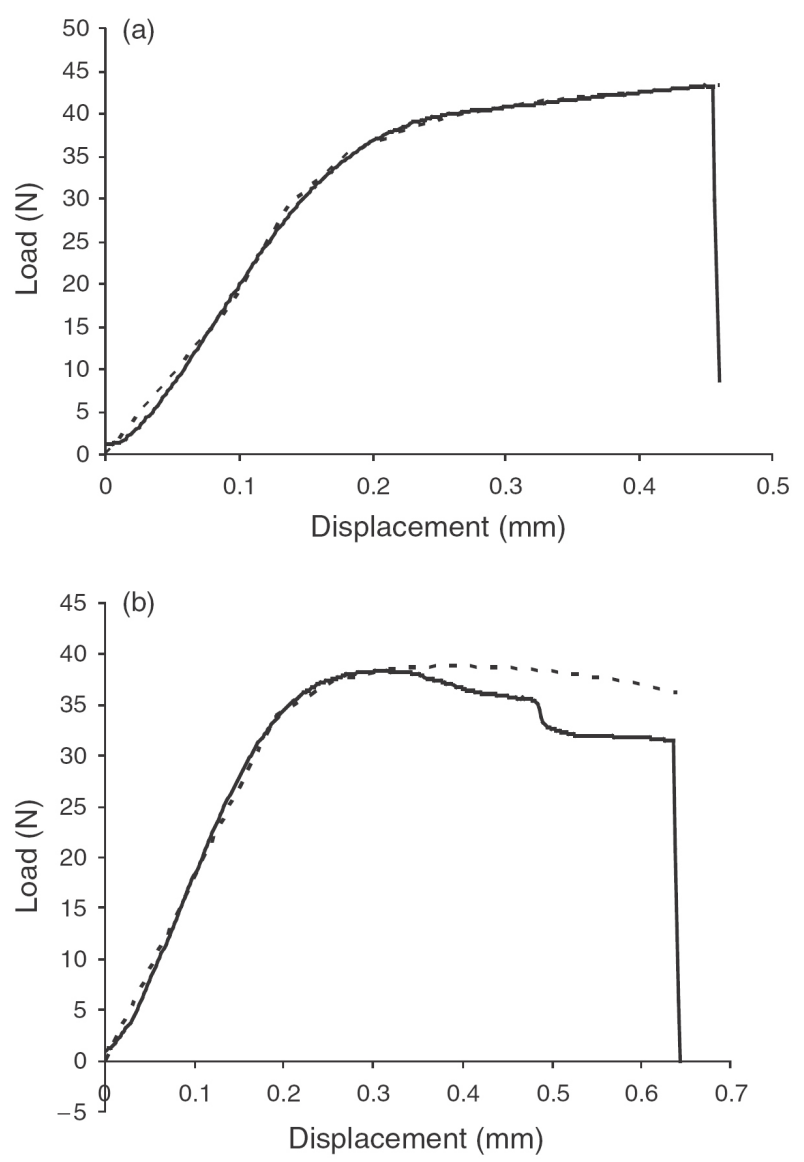

Figure 4. The displacement to load relationship for a column of coyote dentine undergoing three point breaking (a). Superimposed upon it as a dashed line is the displacement to load curve from FEA on a similar sized beam. Using a bilinear model approach, values of $E_{,} E_{t}$, and yield strength are adjusted until a good fit is obtained (fit by eye). (b) One of the few examples where the bilinear model significantly failed to fit the real data. This sample was from an older coyote that produced two other columns that had similar, oddly shaped curves. We assume there was a major flaw in the tooth. Superimposed as a dashed line is our best fit curve from the FEA. 
to force curve to the real curve by eye (Figure 4a) and obtain estimates of the three parameters. These fitted estimates are used to determine the critical stress and strain that occurred at the breaking point. This is done by finding the maximal tensile stress and strain under bending in the sample at the indenter displacement where the column failed. All material properties found by this method are relevant only to bending. When the ultimate stress is calculated for a sample, it is referred to as an ultimate bending stress (UBS) and not the UTS that typically would be calculated from a simple tension test. Likewise Young's modulus and post-yield modulus calculated in bending are relevant only to bending situations.

Once the material properties for a sample were calculated, an analysis was run on a hypothetical standard sample (column $1 \mathrm{~mm}^{2}$ in cross-section). This standardization was needed because of small differences in the cross-sections of columns. Strength and flexibility results from this mathematical analysis can be used to compare samples directly.

\section{FEA of composite structures}

To test the impact of gradients in the material properties of dentine, we built virtual composite beams within our
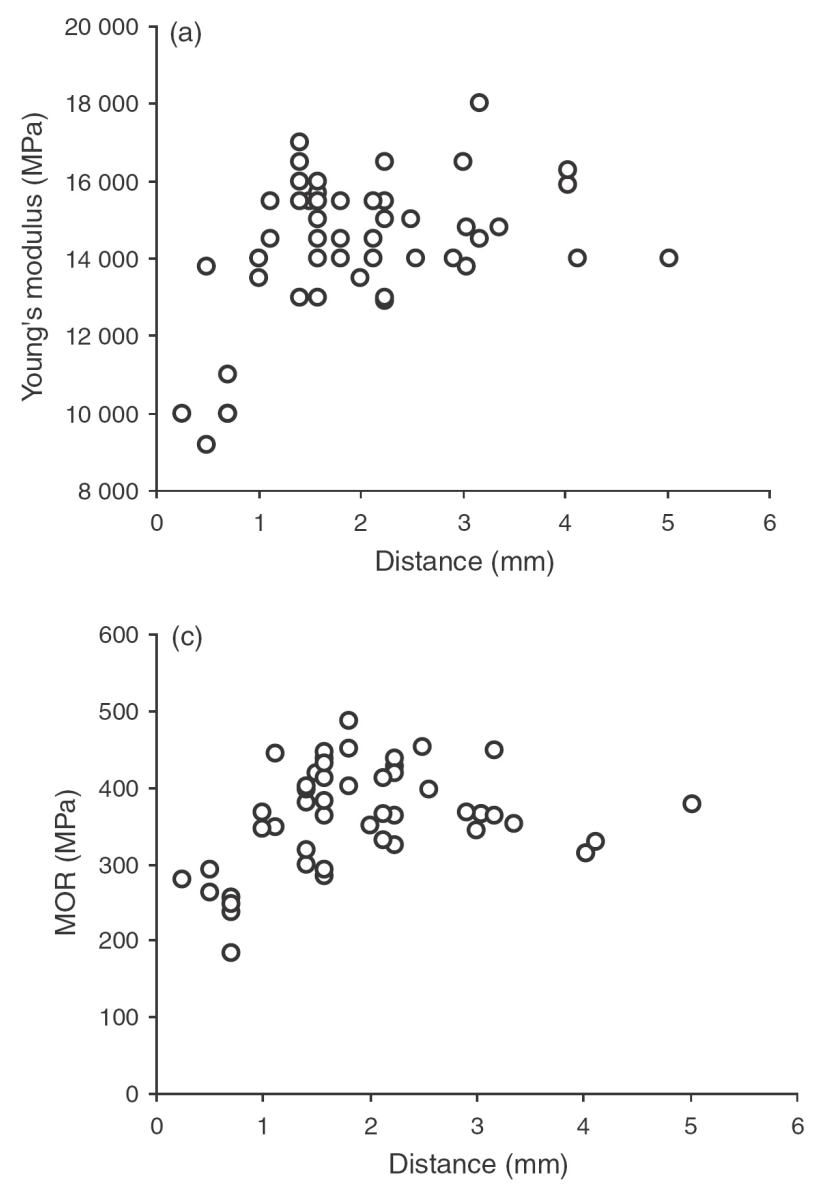

FEA. These model beams had a depth of $3.5 \mathrm{~mm}$ composed of 14 layers, each $0.25 \mathrm{~mm}$ thick. Each layer could be assigned different material properties. The idea was to create a model beam that could simulate the gradients found in real teeth. We used the properties of three extreme samples of dentine that were taken from properties of dentine found in one tooth (Figure 5). To create a beam that reflected the gradients we found in coyote teeth, we assigned highly ductile properties to the outermost layer of dentine $(E=14000 \mathrm{MPa}$, yield strength $=202 \mathrm{MPa}$, post-yield modulus $=436 \mathrm{MPa}$ ). Deeper layers were linearly interpolated to a strong dentine layer $(E=14000 \mathrm{MPa}$, yield strength $=$ $305 \mathrm{MPa}$, post-yield modulus $=260 \mathrm{MPa}$ ) found at layer 6 . In turn the properties of the strong dentine were graded to a dentine of intermediate strength ( $E=15500 \mathrm{MPa}$, yield strength $=270 \mathrm{MPa}$, post-yield modulus $=299 \mathrm{MPa})$ at layer 11 that is typical of dentine found deeper in the tooth. We refer to this model as the composite beam. For comparisons, virtual beams were also constructed where all 14 layers were assigned the properties of the most ductile dentine and another with all layers assigned the properties of the strongest dentine.

Within FEA the model beams were fixed at one end as a simple cantilevered beam. A static load was placed at the other end of the column at the $18 \mathrm{~mm}$ point on the upper
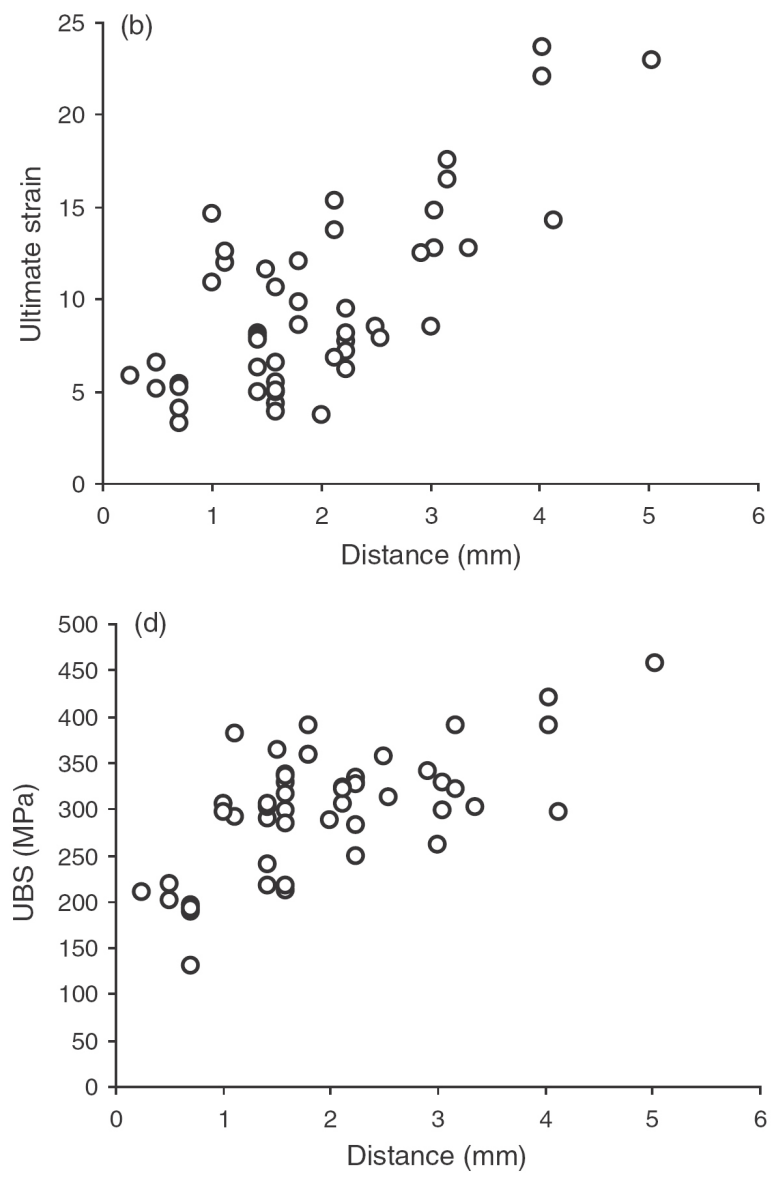

Figure 5. Young's modulus (a), ultimate strain (b), modulus of rupture (c), and ultimate bending stress (d) are plotted against distance from the pulp cavity of the upper canine of a coyote. In all cases there are significant linear and/or quadratic relationships (see text). 
surface of the column. The load was increased until some dentine in the tooth reached its critical strain point.

\section{Results}

\section{A metric for dentine strength}

Ultimate stress can be calculated in the form of either the linear MOR or the bilinear UBS from FEA. The virtue of MOR is its simplicity, and it is perfectly correlated with $P_{\max }$ when using columns of the same size [equation (1)]. No complex FEA analysis is needed. Freeman \& Lemen (2007) have shown that it is effective in predicting the breaking strength of teeth. However, because of nonlinearities in dentine, MOR will not accurately reflect true stresses. UBS from a bilinear FEA will be a more accurate measure of the true stress, but requires a far more complicated analysis. Thus there are cases where the simpler MOR is a good option and is used in some industries (Hoadley, 2000; Callister, 2006). In other cases where a more detailed understanding of the dentine is needed, the nonlinear approach will be needed. There are gradients in the properties of dentine within a tooth, not only in ultimate stress but in other material properties as well. As we try to extrapolate from the properties of a small sample of dentine to the whole tooth, the FEA using a bilinear model must incorporate the composite nature of the strength of dentine with its yield stress, ultimate strain, post-yield modulus, and Young's modulus.

MOR and UBS are shown in Figure 6. As expected most MOR values are higher than the corresponding UBS value (Burstein et al., 1972). Only three samples had UBS greater than MOR. These three samples were the most plastic. In

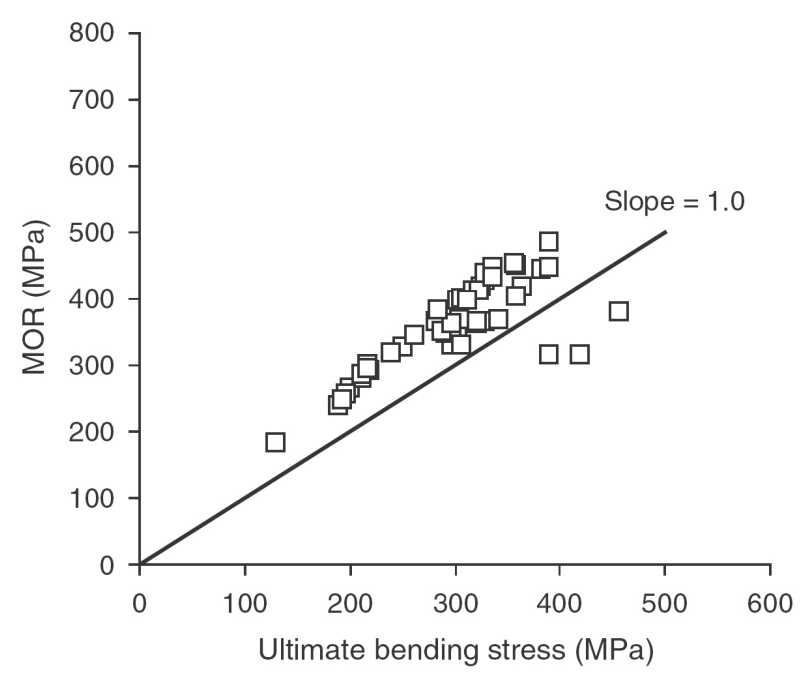

Figure 6. Relationship of UBS (calculated by bilinear FEA) and MOR (calculated from beam theory with a linear assumption). Note most points lie above the slope $=1.0$ line, indicating that MOR is typically higher than UBS. However in more ductile samples stress is predicted to be concentrated in a plastic hinge and UBS may exceed MOR (three points below the slope $=1.0$ line).
FEA these samples form plastic hinges where high stress values are concentrated in a small volume to the extent that the values of UBS exceed MOR. Observation of the ductile dentine just before breaking confirmed the formation of a plastic hinge where strain was concentrated and the dentine in the hinge "whitened" (Currey, 2002).

\section{Importance of position}

We looked at the effect position had on four properties of dentine: Young's modulus, MOR, UBS, and ultimate strain. For Young's modulus the samples closest to the pulp had lower elasticity, but $E$ quickly reached an asymptote (Figure 5a). The MOR showed that dentine near the pulp cavity is weak with strength rapidly increasing with distance (Figure 5c). However, at greater distances MOR drops. A quadratic regression was fit to this relationship and is highly significant $(\mathrm{MOR}=227.7+122.1 \times$ distance $-20.6 \times$ distance $^{2}, F=13.8$, d.f. $\left.=2,46, P<0.0001\right)$. Using a likelihood ratio test (Clark, 2007) to compare this quadratic model to the nested linear model, we found a deviance of $14.47(P<$ 0.001 ), confirming our preference for the quadratic model.

As can be seen in Figure 5b, the relationship between distance and ultimate strain is monotonically increasing. We modeled this relationship with both a quadratic and a linear model. However, in this case the deviance of the likelihood ratio is only $1.34(P>0.20)$, which does not support the selection of the quadratic model over the linear model $(\mathrm{UBS}=220+40.4 \times$ distance, $F=33.45$, d.f. $=1,48, P$ $<0.000001)$.

The difference in ultimate strength and strain can be illustrated by looking at the force to displacement curves

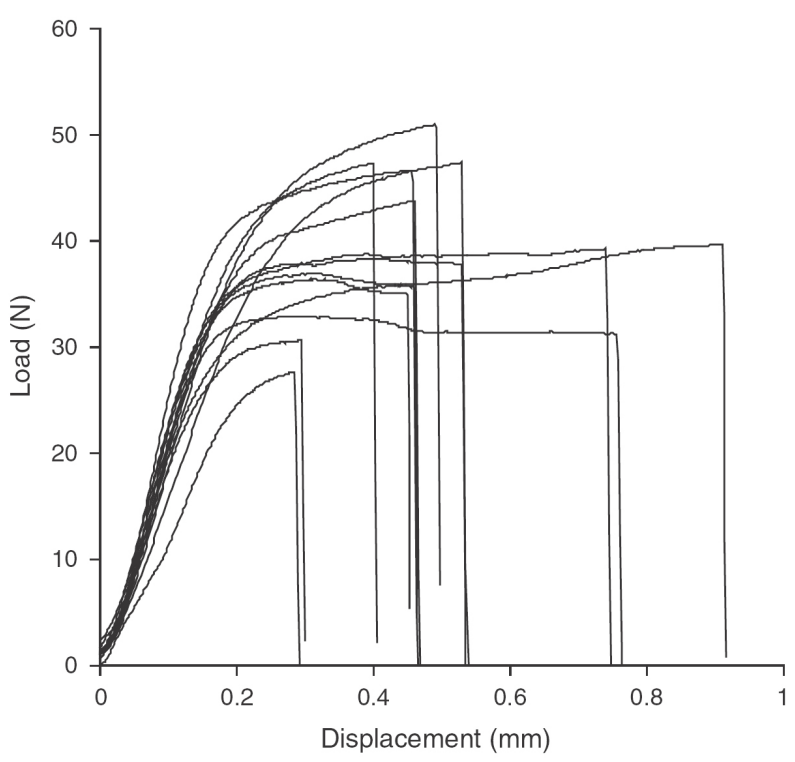

Figure 7. The displacement to load curves for the columns from one canine. As can be seen there is considerable diversity in these curves, which reflects the diversity of dentine in a single tooth. 
for standardized dentine samples (Figure 7). The strongest samples have the highest loads but tend not to be as ductile. The most ductile samples do not have the highest strength.

\section{Anisotropy}

We suspect that coyote dentine is an anisotropic material. Columns that run parallel to the palate are about $50 \%$ weaker than columns that run along the long axis of the tooth. This is consistent with findings for human dentine (Craig, Peyton \& Johnson, 1961; Bowen \& Rodriguez, 1962; Sano et al., 1994). However, because of the small size of the coyote tooth, our cross-sectional columns had to come close to the weaker dentine near the pulp cavity. The columns had about the strength expected of dentine near the pulp cavity with an average MOR of $199 \mathrm{MPa}$. Therefore while we expect that dentine is anisotropic, our data cannot be used to confirm this.

\section{Human results}

The main reason we tested human dentine is because of the large difference in the UBS values we found for carnivores (Freeman \& Lemen, 2007) and published human values. One possibility is that using a rupture test versus a standard tensile strength test could explain the difference. The values of MOR for three columns of human dentine in bending are 215, 234, and $239 \mathrm{MPa}$. These MOR values are higher than the UTS values from the literature (Craig \& Peyton, 1958; Bowen \& Rodriguez, 1962; Sano et al., 1994), as expected (Burstein et al., 1972). The UBS values for three columns of human dentine in bending are 157, 171, and $176 \mathrm{MPa}$. If $125 \mathrm{MPa}$ is taken as the UTS for human dentine then the average ratio of MOR to UTS is 1.8, similar to values found by Burstein et al. (1972) and our results for coyotes. Importantly these human MOR values are still be-

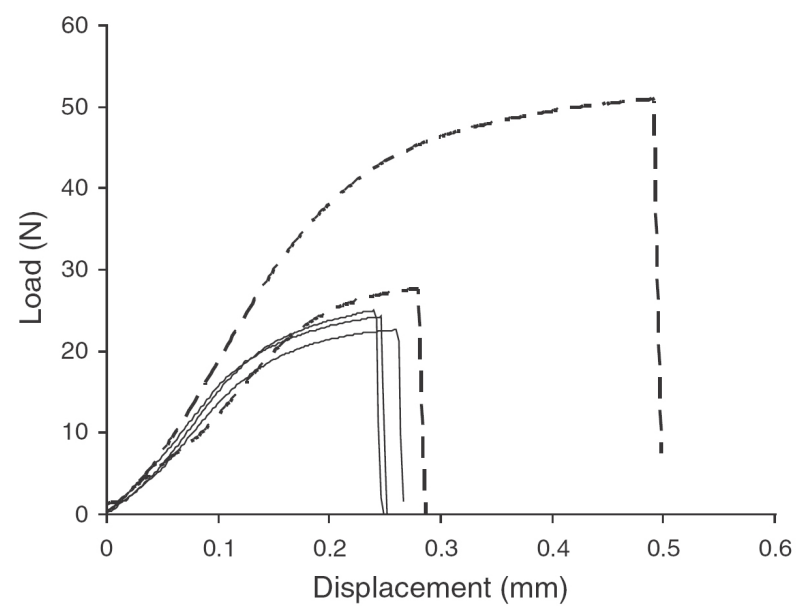

Figure 8. The displacement to load curves for three columns of human dentine are shown as solid lines. Dashed lines show the load to displacement curves for a strong sample of coyote dentine and a very weak sample near the pulp cavity. All columns are of the same size and shape. low average values found in coyotes and are more consistent with the weakest dentine found in coyotes (Figure 8). It will be interesting to see whether there are consistent differences in the properties of dentine across species with different feeding habits, such as humans and coyotes.

\section{Hysteresis and damage}

Dentine, like other viscoeleastic materials, shows hysteresis, and the loading and unloading curves are not the same (Figure 9). Also once the dentine is stressed into plastic deformation beyond the linear elastic zone, damage is created in the tooth that alters the displacement to force curve.

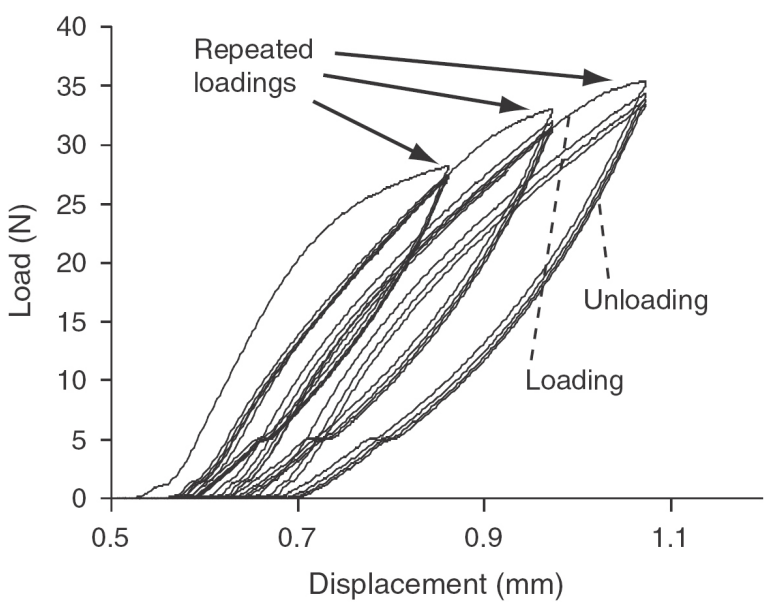

Figure 9. Repeated loading and unloading of a single column of dentine. Hysteresis is revealed by the difference in the shape of the loading and unloading curves. The damage done to the column by loading beyond the linear response point is shown by the progressive shifts of the curves after loading to plastic deformation.

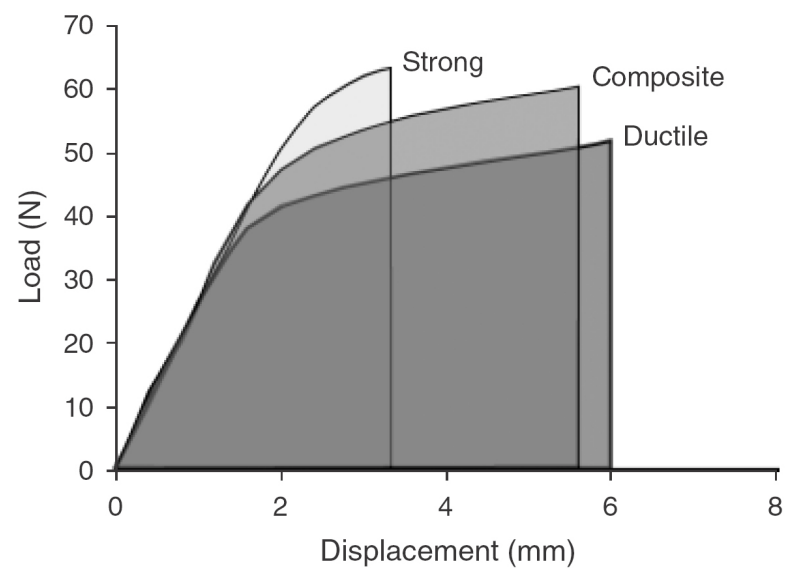

Figure 10. The displacement to load curves for strong-brittle, weak-ductile, and composite beams from FEA. Note that the composite beam has almost the same strength as the strong beam and almost the same ductility as the ductile beam. The composite beam would require the most work of fracture (area under the curve) to break. 
As expected, the maximum stress the dentine can resist remains the same, but the work needed to arrive at this stress is reduced considerably.

\section{Tension and compression test results}

Results from our tensile tests for the coyote are limited; only two columns were successfully broken in tension. These columns were paired with columns from the same tooth in a similar position from the pulp cavity. These mirror columns were broken in bending to obtain MOR values. For the first pair, the column under tension produced a UTS of $195 \mathrm{MPa}$ while its mate under bending produced an MOR of $341 \mathrm{MPa}$ and a UBS of $369 \mathrm{MPa}$. For the second pair, UTS = $225 \mathrm{MPa}, \mathrm{MOR}=406 \mathrm{MPa}$ and UBS $=383 \mathrm{MPa}$. The average of the ratio of MOR to UTS was 1.8, which is about what would be expected from experimental work on bone (Burstein et al., 1972) and our results from human dentine. However, the UTS values are considerably higher than the published values for human dentine (up to about $125 \mathrm{MPa}$ ). This offers further support for the idea that coyote dentine is considerably stronger that human dentine.

Results from compression tests on coyotes resulted in an average compressive strength of $326 \mathrm{MPa}(\mathrm{sd}=42, n=$ 18). This average is similar to the compressive strengths found in human dentine (297 MPa; Craig \& Peyton, 1958). Unlike human dentine, coyote dentine has similar compressive and bending strengths.

\section{Impact of gradients}

Results from testing virtual beams with a realistic distribution of material properties against beams of uniformly strong or ductile dentine are shown in Figure 10. The beam of pure strong dentine was the strongest; however MOR was only $5 \%$ higher than the composite beam. The composite beam had the highest work of fracture.

\section{Whole tooth breaks and half, full grinds}

The important role that enamel plays in teeth is to resist wear. One way to test the role enamel may play in bending strength is to grind the enamel away from the tooth and test tooth strength. One problem with grinding the tooth is that, even with great care, some dentine will be ground off as well as enamel. The force needed to break the teeth is shown in Figure 11 for a paired comparison. For teeth with half grinds there was no weakening. The average strength of half ground teeth was $105 \%$ of whole teeth. Removing just the posterior thin shell of enamel was motivated by the fact that all teeth we studied had a series of hairline factures along the posterior edge of the tooth (Freeman \& Lemen, 2007). It appeared to us that the brittle enamel readily failed in tension and was unlikely to add much strength to the tooth. Our results confirm this hypothesis (Figure 11). Further, the lack of difference in strength between paired, ground and unground, left and right canines supports the notion that grinding can be done without introducing grinding artifacts that weaken the tooth.

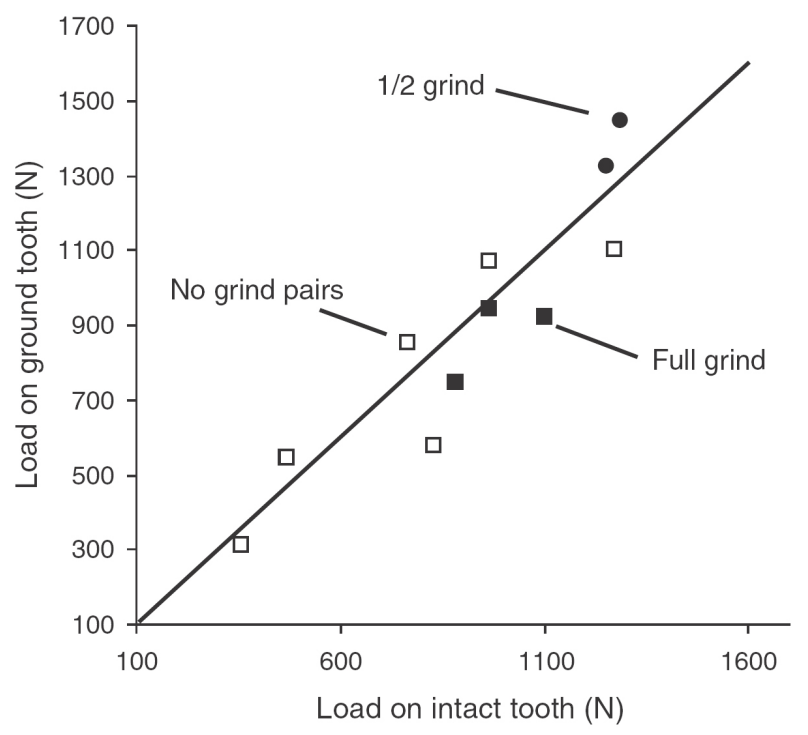

Figure 11. Each symbol represents a pair of teeth. The solid symbols represent the MOR for a pair of canines, one ground and the other intact, from a single coyote. The solid squares are full grinds - intact pairs where all the enamel was removed from the ground tooth. The solid circles represent $1 / 2$ grinds-intact pairs where the enamel was removed from the posterior half of the tooth. The open squares represent pairs of canines from the same animal where neither tooth is ground. Grinding away enamel has little impact on the strength of the tooth.

Next we tested the full grinds where the entire shell of enamel is removed from one of the paired canines. With a small sample size it is not surprising that the paired $t$-test was not significant $(t=2.4, P>0.07)$. However, the ground teeth average only $88 \%$ of the strength of intact teeth.

To find how much the grinding should weaken a tooth, we created models of canines within the FEA that had the full enamel-dentine structure, the posterior half of the enamel removed and all the enamel removed. The dentine and enamel were treated as a single material and modeled as the strongest dentine found in a mature coyote. In the lab, the teeth with all the enamel removed averaged $12 \%$ weaker than the intact teeth, but the FEA predicted they should be $23 \%$ weaker. We concluded that enamel in these teeth at most plays a small role in overall bending strength.

\section{Predictive value of a bilinear model}

Freeman \& Lemen (2007) quantified the relationship between size of tooth and the load needed to break it using a linear model of dentine. Here we are quantifying nonlinear properties of small portions of the tooth (the $1 \times 1 \times 6.36 \mathrm{~mm}$ columns) and extrapolating to predict whole tooth behavior. FEA indicates that realistic gradients of strong and ductile dentine result in overall strength about $5 \%$ weaker than pure strong dentine alone (Figure 10). A real tooth was broken in the lab with a force of 1069 N (Freeman \& Lemen, 2007). FEA of a model of this tooth using the bilinear model of strong dentine predicted an ultimate load of 1116 N. For 
an estimate of the load needed to break a tooth with a realistic gradient in dentine properties, this load is reduced by $5 \%$ to $1060 \mathrm{~N}$.

\section{Discussion}

We found (Freeman \& Lemen, 2007) that the simplifying assumption of linearity and homogeneity would yield MOR values that are useful for predicting the failure of teeth under static load. However, this MOR value is not actually experienced by the tooth and is simply a mathematical construct. Understanding the properties of dentine more thoroughly requires a nonlinear approach and allowance for gradients of properties within the tooth.

In this study we concentrated on failure in bending. We found that UTS determined in a tension experiment is not useful for this task, and we must use data from bending experiments to make predictions about failure in bending. As Currey (2002) warns, there are problems with quantifying stresses and strains during bending. However, we had little choice here. Our comparison of UTS, MOR, and UBS indicates that the linear MOR value and the bilinear UBS values are nearly twice the UTS value. Some (Staninec et al., 2002) have suggested that fracture mechanics may offer a way forward to connect these values and resolve this discrepancy, but that line of investigation is beyond the scope of this paper.

Just as Staninec et al. (2002) found a gradient in dentine's properties across a human tooth, we found a gradient across coyote canines. The weakest dentine is nearest the pulp cavity. This is not surprising because dentine in this position has little to do with overall strength of the tooth in bending (Freeman \& Lemen, 2007). More surprisingly, there is not a simple increase in strength (MOR) of dentine with distance from the pulp cavity (Figure 5). The relationship is curvilinear with a decline in strength at the greatest distances. This appears to be exactly the wrong way to build a strong tooth. Failure of this weaker, outermost dentine will start a crack that quickly causes the whole tooth to break. However, factoring in the ductile nature of this dentine may clarify the situation. There is a strong relationship between distance from pulp cavity and ultimate strain. Initially dentine is getting stronger and more ductile moving away from the pulp cavity. However, at the greatest distances strength begins to fall while ductility continues to rise (Figures 5 and 7 ). When a virtual composite beam with this gradient in properties is constructed in FEA, we found that the virtual beam was nearly the same strength as a beam made of the strongest dentine, but it also has nearly the flexibility of a beam made from the most flexible dentine (Figure 10).

In what way might this gradient of ductility in dentine be an advantage to the coyote? There would be no advantage for static loads. The composite beam breaks at $95 \%$ of the load of the strong beam (Figure 10). For dynamic loading where work of fracture is a key factor, the expectation is that the flexible dentine, with its higher work of fracture, would be at an advantage (Vincent, 1982). In our model this was not the case. While the pure ductile beam had a 1.7 times higher work of fracture than the strong beam, the composite beam required 1.2 times more work to break than the ductile beam (Figure 10). Thus there may be an adaptive explanation to the gradient in dentine. Research is needed to elucidate the gradient in properties at a finer level than was possible in our study. One problem that should be kept in mind is that the ductility of the dentine is bought at the price of damage, probably in the form of microcracks (Currey, 2002). Once this damage is done the tooth has been seriously weakened, not in ultimate load but in work needed to fracture (Figure 9). As there is no evidence for the repair of these microcracks in dentine as there is in bone, once the damage is done, it may be permanent.

One of our goals was to develop a model of tooth strength based on the nonlinear material properties of dentine and the gradients of these properties. We determined that enamel plays a small part at most in tooth strength, with the result that we will use a dentine-only approach to modeling tooth strength. Using a bilinear model, we estimate the material properties of dentine and build an FEA model around a tooth previously broken at $1069 \mathrm{~N}$. The composite model of dentine predicts a maximum load of $1060 \mathrm{~N}$. This close agreement of experiment and simulation is likely part luck. However, it bodes well for the approach of using bending strength and considering the gradient properties of dentine when assessing strength in bending a tooth.

\section{Acknowledgements}

We thank the University of Nebraska Agricultural Research Division, the School of Natural Resources, and the University of Nebraska State Museum for support. We are especially grateful to Mark W. Beatty, Professor, and Bobby Simetich, Research Technologist, Biomaterials Section, College of Dentistry, University of Nebraska Medical Center, for the gracious use of their equipment and time.

\section{References}

Bowen, R. L., and Rodriguez, M. S. (1962). Tensile strength and modulus of elasticity of tooth structure and several restorative materials. J. Am. Dent. Assoc. 64, 378-387.

Burstein, A. H., Currey, J. D., Frankel, V. H., and Reilly, D.T. (1972). The ultimate properties of bone tissue: the effects of yielding. J. Biomech. 5, 35-44.

Callister, W. D. (2006). Materials science and engineering: an introduction. New York: John Wiley \& Sons Inc.

Clark, J. S. (2007). Models for ecological data: an introduction. Princeton: Princeton University Press: pp 144-147.

Craig, R. G., and Peyton, F. A. (1958). Elastic and mechanical properties of human dentine. J. Dent. Res. 37, 710-718.

Craig, R. G., Peyton, F. A., and Johnson, D. W. (1961). Compressive properties of enamel, dental cements, and gold. J. Dent. Res. 40, 936-945. 
Currey, J. D. (2002). Bones: structures and mechanics. Princeton: Princeton University Press.

Freeman, P. W., and Lemen, C. A. (2007). An experimental approach to quantifying strength of canine teeth. J. Zool. (Lond.) 271, 162-169.

Hoadley, R. B. (2000). Understanding wood: a craftman's guide to wood technology. Newton, CT: The Taunton Press.

Imbeni, V., Kruzic, J. J., Marshall, G. W., Marshall, S. J., and Ritchie, R. O. (2005). The dentine-enamel junction and the fracture of human teeth. Nat. Mater. 4, 229-232.

Kishen, A., Ramamurty, U., and Asundi, A. (2000). Experimental studies on the nature of property gradients in the human dentine. J. Biomed. Mater. Res. 51, 650-659.
Sano, H., Ciucchi, B., Matthews, W. G., and Pashley, D. H. (1994). Tensile properties of mineralized and demineralized human and bovine dentine. J. Dent. Res. 73, 1205-1211.

Staninec, M., Marshall, G. W., Hilton, J. F., Pashley, D. H., Gansky, S. A., Marshall, S. J., and Kinney, J. H. (2002). Ultimate tensile strength of dentine: evidence for a damage mechanics approach to dentine failure. J. Biomed. Mater. Res. 63, 342-345.

Van Valkenburgh, B. (1988). Incidence of tooth breakage among large, predatory mammals. Am. Nat. 131, 291-302.

Vincent, J. F. V. (1982). Structural biomaterials. New York: John Wiley \& Sons. 